\title{
Primary angiitis of the central nervous system: 2 atypical cases
}

\author{
Fabio Pagni ${ }^{1}$, Giuseppe Isimbaldi', Francesco Vergani², Paolo Casiraghi², Laura Marzorati ${ }^{3}$, Guglielmo Migliorino ${ }^{4}$, \\ Giorgio Cattoretti ${ }^{1}$ \\ ${ }^{1}$ Department of Pathology, Università Milano Bicocca, San Gerardo Hospital, Monza, Italy, ${ }^{2}$ Neurosurgery Unit, Università Milano \\ Bicocca, San Gerardo Hospital, Monza, Italy, ${ }^{3}$ Neurology Unit, Università Milano Bicocca, San Gerardo Hospital, Monza, Italy, \\ ${ }^{4}$ Infectious Disease Unit, Università Milano Bicocca, San Gerardo Hospital, Monza, Italy
}

\begin{abstract}
The presence of an angiitis process in the central nervous system (CNS) characterizes different groups of conditions: from idiopathic pachymeningitis to lymphoproliferative disorders. In absence of specific infections, inflammatory and neoplastic diseases, the term "PACNS" (Primary Angiitis of the CNS) was proposed to indicate a peculiar vascular inflammation of unknown origin of meningeal vessels extending to the brain or spinal cord parenchyma. We report two cases of PACNS with peculiar and atypical features: the first one with a possible Epstein Barr virus (EBV) relationship, the second one with spinal cord involvement only, treated surgically. We also hypothesize a correlation between EBV chronic infection and possible subtypes of PACNS stressing the importance of EBER (EBV-encoded RNA) test in the routine examination of brain biopsies suspicious for PACNS.
\end{abstract}

Key words: PACNS, granulomatous angïtis, EBV-related disorders.

\section{Introduction}

Primary angiitis of the central nervous system (PACNS) is a rare inflammatory condition, limited to small meningeal vessels (arterioles and venules of 200 micron-calibre) [17] and characterized by the presence of angiotropic inflammation of the meninges and involvement of the deepening branches $[1,20]$ as evolution of the primary process. PACNS mostly affects the cerebral parenchyma. A spinal cord involvement has been reported in only $17 \%$ of cases [21]. The largest review in medical literature describes 101 cases from the Mayo Clinic Archives during a 21-year period, only part of which had a histological confirmation, which should be the gold standard for diagnosis $[2,18]$. The incidence of the disease is 2 per 1000000 . The aetiology is unknown: autoimmunity against a possible viral infection has been proposed [6,12]. Actually to fulfil diagnostic criteria of PACNS specific systemic infections and inflammatory diseases have to be excluded. The differential diagnosis of PACNS includes other cerebral vasculitis (Horton and Takayasu's disease), herpetic angiitis, drug-related vasculitis (cocaine), other inflammatory processes (neurosarcoidosis - NS) and neoplastic conditions (lymphomatoid granulomatosis - LG, vanishing lymphoma syndrome). A possible etiologic viral role has been proposed for many of these entities; especially Epstein-Barr virus (EBV) could be involved in vasculitis such as leukocytoclastic angiitis, panarteritis nodosa, lupus-associated vasculitis but also in demyelinating encephalopathies (multiple sclerosis - MS) [22], Guillain-Barrè syndrome and malignant lymphomas (EBV associated lymphoproliferative disorders). 


\section{Case reports}

\section{Case 1}

A 31-year-old Philippine patient was admitted to the Neurosurgical Department of our Institution, with a 3-4 months' history of persistent nycturia, urinary incontinence and asthenia. Magnetic resonance imaging (MRI) showed a strong leptomeningeal enhancement around the encephalic trunk and lumbar spinal cord (Fig. 1, upper left). The cerebrospinal fluid (CSF) analysis showed high protein levels, low glucose; the result of CSF sedimentation assessment was 228 mononuclear cells $/ \mathrm{mm}^{3}$, essentially lymphocytes; Cyto- megalovirus, Herpes Simplex Virus 1-2 serum tests were negative. Empiric anti-tubercular (TBC) therapy with Isoniazid was planned but the test for MycobacteriumDNA was negative by semiquantitative Polymerase chain reaction (PCR). The clinical and radiologic findings could be compatible with the diagnosis of NS, meningeal leukaemia or lymphoma. The neurological status was rapidly worsening: the vertical position was kept with difficulty because of spasticity and myoclonus. A second CSF analysis showed the presence of significant (> $40 \mathrm{cp} / \mathrm{ml}$ ) copies of EBV-DNA by PCR. No signs of EBV acute or chronic systemic infection or immune deficiency were present. EBV re-activation with-



Fig. 1. Case 1. Upper left: MRI shows a leptomeningeal enhancement around encephalic trunk and lumbar spinal cord. On the right: autopsy brain sections; the upper images show ischemic lesions within internal capsule, caudate and lentiform nucleus (arrow); in the lower section the arrow indicates the results of the ventricular catheter. In the upper right, histology reveals spread ischemic features (neuronal necrosis, accumulation of foam cells, oedema). Inset: clear-cut angiitis in meningeal spaces. Inner left: lymphoid perivascular infiltrate composed of a polymorphous population with scattered giant cells. Immunohistochemistry underlines T-lymphocytes (CD3+) in the perivascular sheets; these cells show focal reactivity for cytotoxic antigen (Perforin). Inner right: the EBER analysis shows rare positive cells (arrows). 
in a CNS-limited lymphoproliferative disorder was suspected for the presence of oligoclonal bands in the CSF and a steroid therapy began (methylprednisolone pulse $1 \mathrm{~g} /$ day, then $2 \mathrm{~g} /$ day). After a transitory improvement, the patient experienced seizures. A final tetraventricular hydrocephalus was treated with peritoneal derivation. The patient died 62 days after admission. An autopsy was performed.

\section{Case 2}

A 24-year-old woman was referred to our Centre because of neck pain with irradiation to the right arm, ipsilateral lower limb weakness and diffuse paresthesias. Spinal MRI showed an intramedullary C5-D1 lesion, with strong enhancement after gadolinium, suggestive of a tumour (Fig. 2, lower left and right). Cerebral MRI was negative. Several serum examinations show- ed no bacterial or viral infections; serum EBV-detection was negative by PCR and no oligoclonal bands were detected in a spinal tap sample. CSF flow cytometry did not reveal any significant clonal lymphoid population. Laboratory tests did not reveal any evidence of metabolic, autoimmune or infectious disorder (in particular, NS and TBC were excluded). A surgical indication was given. At surgery, the medulla appeared swollen and brownish; a $5 \mathrm{~cm}$-maximum diameter lesion was completely removed. The histological examination excluded the neoplastic nature of the mass. The final diagnosis of PACNS with unique spinal localization was made. No medical treatment was administered after the surgical excision and a strong follow-up was planned. The postoperative neurologic examination showed the appearance of mild hemiparesis on the right side and a level of hypoesthesia. Motor deficits had a marked improvement after rehabilitation. The pa-

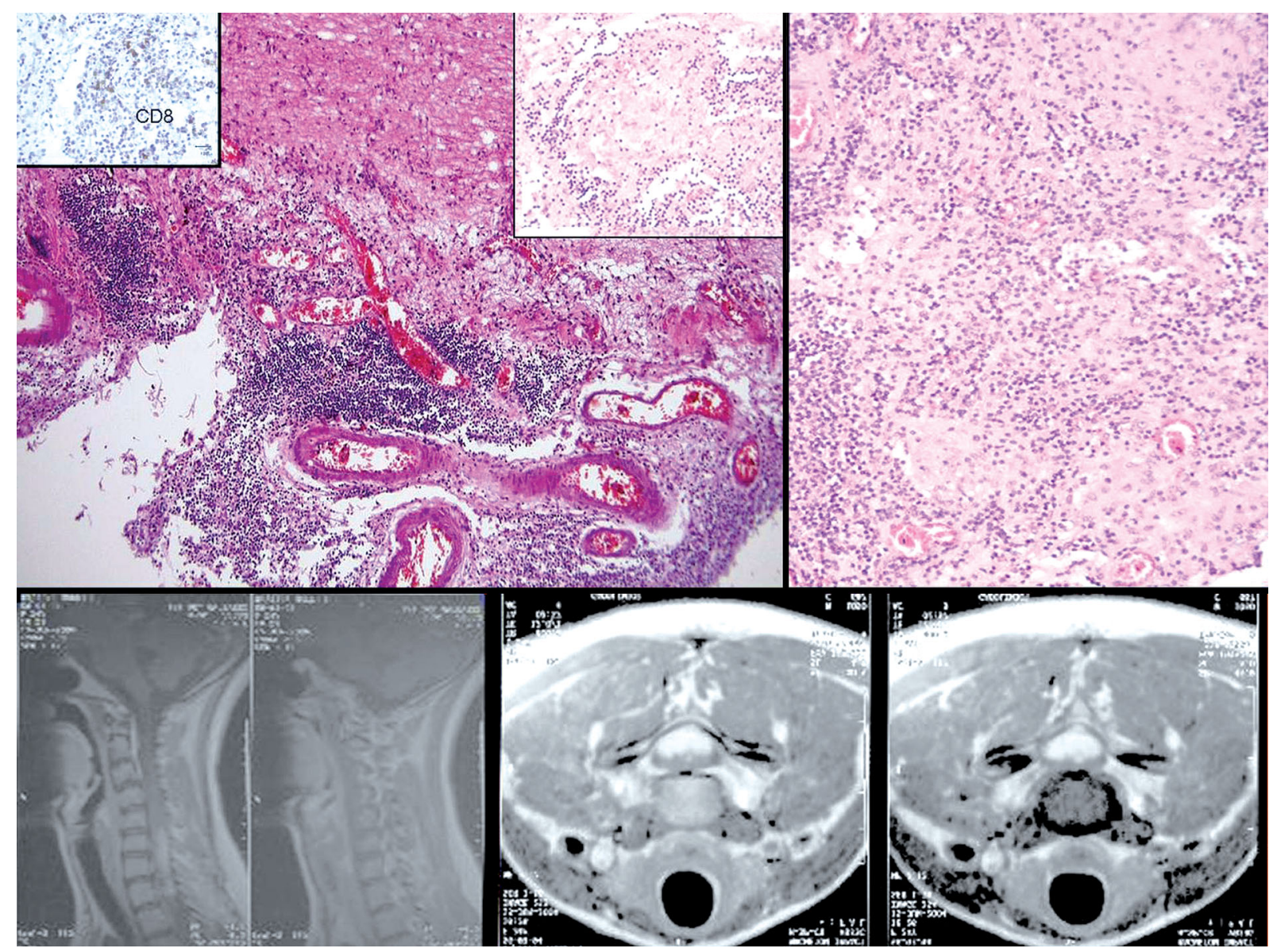

Fig. 2. Case 2. MRI shows an intramedullary lesion in the dorsal and cervical tract (C5-D1) with the cystic cavity in the bulbar region. Histology revealed granulomatous features with a vasculitic component of lymphocytes. Positivity for CD8 was evident in T lymphocytes. 
tient is alive and well without any neurological signs of brain involvement 5 years later.

\section{Pathological findings}

Buffered formalin-fixed, paraffin-embedded tissue was obtained from CNS (brain, meninges and spine) specimens. $3 \mu \mathrm{m}$ sections were cut for Haematoxylin \& Eosin (H\&E) and special stains. Immunohistochemistry was performed on an automated immunostainer (Dako, Glostrup, Denmark) after heat-mediated antigen retrieval. Immunohistochemical studies were performed using antibodies specific of human CD3 (Polyclonal, Dako 42DK-2600 Glostrup, Denmark), CD4 (mouse monoclonal antibody/MoAb 1F6, Novocastra Laboratories, Newcastle, UK), CD8 (MoAb, C8/144B, Dako), CD20 (MoAb L26, Dako), CD79a (MoAb JCBH7, Dako), CD30 (MoAb BER-H2, Dako), CD68 (MoAb PGM1, Dako), Perforin Ab-2 (MoAb 5B10, Neomarkers Thermo Fisher Scientific, Fremont, CA, USA) on specimens and appropriate positive controls. EBV-encoded RNA (EBER) - in situ hybridization was performed using EBV PNA probe (Histosonda, Cenbimo, Lugo, Spain). Immunoglobulin (Ig) heavy chain gene rearrangement studies were performed by PCR.

\section{Case 1}

The cranial cavity displayed smooth meninges. The brain was symmetrical and presented serrated convolutions due to conspicuous oedema. The great vessels and the Willis polygon showed no alterations, with conservation of normal calibres. Sectioning the brain, multiple areas of reduced consistency in the right tha-lamic region and left internal capsule appeared (Fig. 1, upper). Frank hydrocephalus was present. The surgical results of the ventricular derivation were evident in the right lateral ventricle (Fig. 1, upper). At microscopic examination, the brain revealed diffuse ischemic features varying from acute neuronal necrosis to accumulation of granular histiocytic foam cells (Fig. 1, upper right). These ischemic findings were associated to diffuse perivascular oedema with focal haematic congestion. In the cerebral parenchyma, neither lymphoid nodules nor granulomas were noted. At high power, the meningeal samples displayed a focal infiltrate comprising a mononuclear lymphoid component with plasma cells, small polymorphic lymphocytes and histiocytes (Fig. 1, left). This inflammatory infiltrate had a perivascular distribution (venules and small-sized arteries of 30-100 mm diameter); focal fibrinoid necro- sis was evident. Scattered occasional multinucleate giant cells of Langhans-type were present (Fig. 1, middle). The infiltrate consisted of B (CD79a-positive) and T (CD3-positive, CD4-negative) lymphocytes that displayed also CD8-positive elements. Scattered reactivity ( $<5 \%$ of T-cells) for Perforin was evident (Fig. 1, lower left). The monocytic-macrophagic cells were CD68(PGM1) positive, including also giant cells. EBER-in situ hybridization showed some lymphoid cells exhibiting nuclear signal (5-6 cells x 10 HPF, Fig. 1, lower right). No plasma cell clonal restriction was evident by kappa and lambda immunoglobulin light chain staining. Ig heavy chain gene rearrangement (PCR) did not show clonality. The general autopsy excluded any inflammatory or neoplastic systemic process. In particular, no haematological disorder was evident in the bonemarrow biopsies. Moreover, when searching for a possible cause of the urological symptoms, no significant infection of the kidneys and urinary tract was found. A diagnosis of PACNS was rendered, leading to multiple cerebral ischemic lesions, massive oedema and tetraventricular hydrocephalus.

\section{Case 2}

A $5 \mathrm{~cm}$-maximum diameter lesion composed of multiple fragments of grey soft tissue was submitted for histological examination. On microscopic examination, the specimen contained nervous tissue infiltrated by an inflammatory process. No evidence of a neoplastic lesion was present; a mononuclear infiltrate composed of lymphocytes and plasma cells was seen with a perivascular distribution (Fig. 2, upper left) and widespread granulomatous pattern without any evidence of necrosis (Fig. 2, upper right). Immunohistochemical studies revealed the angiotropic infiltrate to be composed of B (CD79a-positive) and T (CD3-positive) cells; there were focal CD8 positive elements (Fig. 2, upper left) and no significant CD4 and Perforin reactivity. Plasma cells did not demonstrate immunoglobulin light chain restriction. CD68 (PGM1) decorates the histiocytes in the granulomas. EBV in situ hybridization was negative. Ziehl-Neelsen staining was negative. The final diagnosis of PACNS with unique spinal localization was performed.

\section{Discussion}

We report two atypical cases of PACNS underlining various possible presentations of this insidious syndrome. Angiitis in the CNS is present in different con- 
ditions: from idiopathic pachymeningitis to lymphoproliferative disorders. The term "PACNS" was proposed to indicate a peculiar vascular inflammation of unknown origin of meningeal vessels extending to the brain or spinal cord parenchyma. This condition is very rare and to fulfil diagnostic criteria of PACNS, specific systemic infections and inflammatory diseases have to be ruled out. We have tried to apply strong clinical and pathologic diagnostic criteria of PACNS for the correct management of the two described cases: first of all excluding other forms of systemic or cerebral vasculitis. Giant cell arteritis (Horton type) is an autoimmune disease in which a primitive and isolate involvement of the CNS is very unusual $[14,20]$. Systemic necrotizing vasculitis like polyarteritis nodosa (a recent case report shows a correlation with EBV [4]) involves medium and small-sized arteries. Veins and capillaries are spared. Because of the granulomatous histological features in our two cases, we then excluded TBC and NS by clinical- pathological and laboratory findings. We have also excluded a lymphoma especially in the autoptic case (Ig heavy chain gene rearrangement by PCR was polyclonal). Finally, in differential diagnosis we had to consider leptomeningeal inflammatory pseudotumor, idiopathic pachymeningitis and inflammatory myofibroblastic tumour. We ruled out these conditions evaluating the absence of the significant fibroblastic component in histological findings with no involvement of dura mater. So a diagnosis of isolated vasculitis of the CNS was possible. These processes are exceedingly rare and do occur mostly in association with a disturbed immune function. There are reports of isolated CNS vasculitis following systemic lupus erythematosus [13], rheumatoid arthritis [15], immunization and X-linked lymphoproliferative (XLP) disease $[8,23]$. In our patients the clinical settings lacked any of these conditions and so the final diagnosis of PACNS was proposed. However, the two presented cases differed from other "classic" PACNS cases and were chosen for this paper for their peculiar features. Even if they could be included in the "PACNS" category, they have some atypical aspects. The autoptic case showed a clinical behaviour completely adherent to the aggressive course of "classic" PACNS. The atypical finding was a possible relationship existing between EBER positivity on the brain tissue and the etiologic development of this PACNS. We did not think that EBER positivity was a sufficient reason for re-classifying the case as EBV-associated angiitis (and not as PACNS). In fact, in the clinical history there were no criteria of an acute or chronic EBV infection. We hypothesized that EBER positivity could be the expression of immunological reactivation of EBV. In this case, the infiltrating lymphocytes were B (EBER-positive/-) and T cells (CD8+/CD4-), although the exact targets of the immune response could not be determined. EBV is a $B$ lymphotropic human DNA herpes virus that causes meningitis, encephalitis, radiculitis [7] but it can also be related to lymphoproliferative disorders and autoimmune processes. Several XLP patients develop CNS vasculitis following systemic EBV activation and lymphoproliferation. In these cases the activation of CD8-positive lymphocytes by EBV-positive B cells lead to the vessel damage; alternatively the vessel walls might themselves become directly infected by EBV [23]. Interestingly, other patients with XLP disease die of EBV negative lymphocytic vasculitis involving the CNS [23]. In these cases, aberrantly activated T cell populations were described in the CNS bearing strikingly similar histological and immunological features to our report. In fact, even in the absence of detectable EBV infection, CD8-positive lymphocytes caused vessel damage with frank vasculitic features probably following a different antigen trigger. The relationship between EBV and vascular damage is well known [11]. EBV-positive malignant T- or B-cell proliferations, angioinvasive cytotoxic T/NK-cell lymphomas or LG are well reported [19]. In the case of $L G$, which we considered in differential diagnosis in the autoptic case, primary or secondary CNS involvement has been described [16]. In primary CNSLG (19 cases in the literature [10]), contrary to systemic LG, EBER test has proven to be negative in all but two HIV-positive cases [10]. Both PACNS and LG present a polymorphous infiltrate in the vascular walls; the prominent angiocentric distribution of lymphoid cells and the absence of eosinophils or giant cells are more consistent with a LG diagnosis than granulomatous vasculitis. Finally, patients with chronic EBV infection have been reported to develop angiitis-driven neuropathy [7] and recently, EBV has been shown in B cell in meningeal and parenchymal lesions in MS [22]. The infection is associated with a CD8-positive cytotoxic response, which may be responsible for the demyelinating process and plaque formation. In some MS cases, very few EBV-positive cells could be detected. Starting to our EBV-associated case, we hypothesize that also in some angiitis of the CNS an immune-mediated vasculitis is driven and fuelled by an angiocentric cytotoxic response to EBV, whereas the pathological mechanism remains unclear. EBV detection in non- 
malignant CNS diseases such as MS has eluded many investigators for a long time, because it is not always easily evaluable. So we propose that EBER test in histological examination suspicious for cerebral angiitis could play an important diagnostic role. We also hypothesize that EBER could represent a prognostic test as in $L G$, where primary cerebral EBV-negative $L G$ appears to run a better course than systemic EBV-positive LG with CNS localization $[9,10]$. The second case was a histology-proven PACNS. However, the clinical setting of the lesion was very atypical. In the literature many PACNS cases have a spinal cord presentation but the subsequent cerebral involvement is the rule [5]. In the patients with spinal cord PACNS the inflammatory process hits the posterior medullary vessels in the thoracic and lumbar segments. The MRI usually shows irregular lesions in the posterior wall of the spinal cord associated to atrophy of the parenchyma. Atypical lesions with tumour-like appearance or an expansive pattern of growth (as our case) are exceptional [3] and seem to be related to a good prognosis and a less aggressive clinical behaviour. A radiological diagnosis of possible neoplastic lesion brought the patient to the surgical theatre. The unique spinal cord localization associated to a good surgical cleavage of the mass permitted the complete excision of the lesion. Histology is the gold standard for the correct diagnosis of these cases in which only the morphological features of the granulomatous inflammatory process can exclude a neoplastic process such as ependymoma. Our neurological team did not perform any medical treatment after the surgical management of this case because the patient did not show any symptoms of CNS involvement and after 5 years is alive and well. Our second case confirms the good prognosis of localized PACNS and proposes the surgical treatment as a possible option for tumour-like lesions with easy surgical access and complete excision outcome.

\section{References}

1. Bellezza G, D’Amico AM, Luthy M, Giansanti M. Primitive angiitis of the central nervous system. An autopsy case report. Pathologica 1998; 90: 459-462.

2. Beppu T, Inoue T, Nishimoto H, Nakamura S, Nakazato Y, Ogasawara K, Ogawa A. Primary granulomatous angiitis of the central nervous system: findings of magnetic resonance spectroscopy and fractional anisotropy in diffusion tensor imaging prior to surgery. Case report. J Neurosurg 2007; 107: 873-877.

3. Bibhatbhan A, Katz NR, Hudon M, Clark AW, Hurlbert RJ, Zochodne DW. Primary angiitis of the spinal cord presenting as a conus mass: long-term remission. Surg Neurol 2006; 66: 622-626.

4. Caldeira T, Meireles C, Cunha F, Valbuena C, Aparício J, Ribeiro A. Systemic polyarteritis nodosa associated with acute Epstein-Barr virus infection. Clin Rheumatol 2007; 26: 1733-1735.

5. Campi A, Benndorf G, Filippi M, Reganati P, Martinelli V, Terreni MR. Primary angiitis of the central nervous system: serial MRI of brain and spinal cord. Neuroradiology 2001; 43: 599-607.

6. Goertz C, Wegner C, Brück W, Berlit P. Primary angiitis of the CNS with pure spinal cord involvement: a case report. J Neurol 2010; 257: 1762-1764.

7. Kanai K, Kuwabara S, Mori M, Arai K, Yamamoto T, Hattori T. Leukocytoclastic - vasculitic neuropathy associated with chronic Epstein-Barr virus infection. Muscle Nerve 2003; 27: 113-116.

8. Kanegane H, Ito Y, Ohshima K, Shichijo T, Tomimasu K, Nomura K, Futatani T, Sumazaki R, Miyawaki T. X-linked lymphoproliferative syndrome presenting with systemic lymphocytic vasculitis. Am J Hematol 2005; 78: 130-133.

9. Kimura H, Ito Y, Suzuki R, Nishiyama Y. Measuring Epstein-Barr virus (EBV) load: the significance and application for each EBVassociated disease. Rev Med Virol 2008; 18: 305-319.

10. Lucantoni C, De Bonis P, Doglietto F, Esposito G, Larocca LM, Mangiola A, Martini M, Papacci F, Teofili L, Pompucci A. Primary cerebral lymphomatoid granulomatosis: report of four cases and literature review. J Neurooncol 2009; 94: 235-242.

11. Maeda E, Akahane M, Kiryu S, Kato N, Yoshikawa T, Hayashi N, Aoki S, Minami M, Uozaki H, Fukayama M, Ohtomo K. Spectrum of Epstein-Barr virus-related diseases: a pictorial review. Jpn J Radiol 2009; 27: 4-19.

12. Miller DV, Salvarani C, Hunder GG, Brown RD, Parisi JE, Christianson TJ, Giannini C. Biopsy findings in primary angiitis of the central nervous system. Am J Surg Pathol 2009; 33: 35-43.

13. Mrabet D, Meddeb N, Ajlani H, Sahli H, Sellami S. Cerebral vasculitis in a patient with rheumatoid arthritis. Joint Bone Spine 2007; 74: 201-204.

14. Myung J, Kim B, Yoon BW, Lee SK, Sung JJ, Chung CK, Chang KH, Park SH. B-cell dominant lymphocytic primary angiitis of the central nervous system: four biopsy-proven cases. Neuropathology 2010; 30: 123-130.

15. Nikolov NP, Smith JA, Patronas NJ, Illei GG. Diagnosis and treatment of vasculitis of the central nervous system in a patient with systemic lupus erythematosus. Nat Clin Pract Rheumatol 2006; 2: 627-633; quiz 634.

16. Nishihara H, Tateishi U, Itoh T, Nagashima K, Tanaka S. Immunohistochemical and gene rearrangement studies of central nervous system lymphomatoid granulomatosis. Neuropathology 2007; 27: 413-418.

17. Paisansinsup T, Manno EM, Moder KG. Cauda Equina Syndrome as a Clinical Presentation of PrimaryAngiitis of the Central Nervous System (PACNS). J Clin Rheumatol 2004; 10: 265-268.

18. Panchal NJ, Niku S, Imbesi SG. Lymphocytic vasculitis mimicking aggressive multifocal cerebral neoplasm: $\mathrm{mr}$ imaging and MR spectroscopic appearance. Am J Neuroradiol 2005; 26: 642-645.

19. Rezk SA, Weiss LM. Epstein-Barr virus-associated lymphoproliferative disorders. Hum Pathol 2007; 38: 1293-1304. 
20. Salvarani C, Brown RD Jr., Calamia KT, Christianson TJ, Huston J 3rd, Meschia JF, Giannini C, Miller DV, Hunder GG. Primary CNS vasculitis with spinal cord involvement. Neurology 2008; 70: 2394-2400.

21. Schmidley JW. 10 questions on central nervous system vasculitis. Neurologist 2008; 14: 138-139.

22. Serafini B, Rosicarelli B, Franciotta D, Magliozzi R, Reynolds R, Cinque P, Andreoni L, Trivedi P, Salvetti M, Faggioni A, Aloisi F. Dysregulated Epstein-Barr virus infection in the multiple sclerosis brain. J Exp Med 2007; 204: 2899-2912.

23. Talaat KR, Rothman JA, Cohen JI, Santi M, Choi JK, Guzman M, Zimmerman R, Nallasamy S, Brucker A, Quezado M, Pittaluga S, Patronas NJ, Klion AD, Nichols KE. Lymphocytic vasculitis involving the central nervous system occurs in patients with X-linked lymphoproliferative disease in the absence of Epstein-Barr virus infection. Pediatr Blood Cancer 2009; 53: 1120-1123. 